\title{
ALAIN RICARD
}

\section{L'EFFET ROMAN DANS LES LANGUES DE L'AFRIQUE QUELQUES RÉFLEXIONS COMPARATISTES}

\begin{abstract}
In 2006 Xavier Garnier and the present author published a collection of essays derived from a three years long monthly seminar held at the CNRS in Paris on the coming of the novel in the languages of Africa (L'effet roman, Paris 2006). They considered the situation in 27 different languages, analyzing it in details in 20 of them. In the present article the author reflects upon the conclusion and prospects of this comparative enterprise. Quite often the production of fiction is seen as a symbol of cultural achievement in a language: little is known about the texts themselves, their messages, their style. Thomas Mofolo is widely held to be the first novelist writing in an African language, but his first novel, Moeti oa Bochabela, has rarely been read in a political and historical perspective. It is the story of questions posed by the coming of Christianity to Africa and this was a truly revolutionary questioning at that time (1907). While diglossia and bilingualism are the rule in Africa, literatures in African languages are too rarely analyzed along with literature in European languages. The perspectives offered by the political content of the concepts of polyphonia and dialogic consciousness, presented by Bakhtin, acutely conscious himself of these dimensions, are not taken with their philological implications in Africa. The novel is a new genre in many European languages, too, and it corresponds with an era of national consciousness. In many African languages careful historical study allows us to reach the same conclusions despite the colonial control, and the recent (second half of the twentieth century) domination of European languages. The paper concludes by a plea for translation, and quotes the recent work of Ngugi wa Thiongo as a new and positive direction for African writers.
\end{abstract}

Quiconque s'intéresse à la littérature et au livre ne peut qu'être frappé par la minceur des titres parus en langues africaines. Non seulement il en paraît peu, mais encore ils dépassent rarement les 200 pages. J'achète depuis plus de 35 ans des livres en Afrique, des livres en prose qui racontent une histoire continue, dans des langues que je ne connais pas. Ils se présentent comme des romans : souvent divisés en chapitres, écrits en prose. A dire vrai, il y en a peu en prose continue, et à de rares exceptions près, sur lesquelles je vais revenir, ils sont assez peu volumineux. Mais tout d'abord, qu'est ce qu'un roman, en général ? Nous reprendrons les termes de Thomas Pavel :

Je suivrai par conséquent l'exemple de ceux qui font un usage informel et coutumier du terme « roman », en y incluant non pas les œuvres qui satisfont une définition préalable, mais plutôt celles qui, au long des siècles, ont été saluées et lues comme des romans (Pavel, 2003 : 44).

Les livres que j'achète sont vendus comme des romans et se présentent comme des romans. Dans ses Essais de littérature (vraiment) générale, en 1974, Etiemble nous entretenait de littérature chinoise mais aussi du roman philippin. Je voudrais approcher avec modestie cette 
réflexion générale en l'appliquant aux livres produits en Afrique, dans des langues de l'Afrique. Robert Escarpit s'intéressait au développement de l'écrit comme support de communication : une faim de lire ne doit pas être la fin du livre. En somme le livre a encore et pour longtemps sa place dans l'horizon de toutes les cultures, et l'écran ne peut le remplacer. Quelle place, alors, les littératures de l'Afrique peuvent-elles avoir dans la mondialisation de la culture ? Ces minces ouvrages, ces romans-brochures écrits dans des langues peu connues et lointaines, pourront-ils un jour être lus ailleurs, connaître une plus large diffusion? Leurs auteurs sont ils condamnés à cesser de s'exprimer? La défense de ces expressions écrites n'est-elle pas une défense de la liberté d'expression, de la variété, de la biodiversité culturelle, qui est aussi l'un des grands ressorts humanistes des études de littérature comparée?

\section{L'ARRIVEE DU ROMAN}

Je viens de publier avec Xavier Garnier un ouvrage intitulé L'Effet roman, produit d'un séminaire de littérature organisé pendant trois ans au sein du laboratoire du CNRS qui travaille sur les langues et les cultures de l'Afrique ${ }^{1}$. Ce volume rassemble les communications d'une vingtaine de chercheurs, portant chacune sur une langue différente. Pour diverses raisons, nous avons renoncé à publier des exposés complémentaires, mais les langues et les littératures considérées en détail sont néanmoins au nombre de 27 et couvrent une grande partie de 1'Afrique. En annexe de cet article figure la liste des langues et les titres des ouvrages étudiés, par ordre chronologique. Il faut noter que ce type de liste est une première. Nous avons établi, pour chaque langue, le titre du premier texte lu comme roman. Dans les cas litigieux, nous avons expliqué les raisons du litige ; nous avons, dans chaque cas, analysé en profondeur le contexte d'écriture, les traits stylistiques, et la réception de chacun de ces livres dont plusieurs sont très célèbres dans leur communauté linguistique, mais totalement inconnus ailleurs, par exemple le texte luganda. Dans de nombreuses langues il existe donc des romans : quel rôle jouent-ils dans leur langue respective en tant qu'objet textuels ? Et que racontent-ils qui ne soit déjà dans la sempiternelle tradition orale, toujours convoquée, voire invoquée pour comprendre les littératures de l'Afrique ? L'arrivée du roman nous a paru provoquer un effet spécifique, qui donne son nom au livre, l'effet roman. Des blocs de prose imprimée signalent le passage de l'histoire de la langue à

\footnotetext{
${ }^{1}$ UMR 8135, Laboratoire LLACAN: Langage, langues et cultures de l'Afrique noire.
} 
un autre registre. Les sociolinguistes parlent d'aménagement linguistique quand ce passage est géré par des spécialistes qui composent dictionnaires, lexiques, rapports, tous outils nécessaires à l'activité littéraire. Mais le passage au roman vient avec ou après l'aménagement. Il est souvent corrélé avec l'augmentation du lectorat, avec la prise en charge de la production textuelle écrite par des groupes culturels ou politiques pour qui la production de la prose, la production d'un roman est signe de réussite culturelle et politique, exemple à suivre, à méditer, à répéter.

Nous avons examiné les réussites de ce type. Nous avons analysé les conditions d'apparition du roman à l'époque coloniale, période dans laquelle de nombreuses langues africaines, pour de bonnes ou de mauvaises raisons, ont été développées. Cette prise en charge, hier comme aujourd'hui, a rarement abouti à de bons textes, n'échappant pas au destin ambigu de la littérature sous tutelle. Pourtant nous avons souhaité relire certains de ces textes, dégager leurs dimensions prophétiques ou utopiques, rarement prises en compte dans le contexte colonial, mais encore vivantes aujourd'hui.

Notre travail est parti de considérations générales qui relèvent de la philologie. Elena Bertoncini, dans un article qui est à l'origine du séminaire, analyse la notion et les procédés syntaxiques et morphologiques qui permettent de signaler le style indirect, éventuellement libre, en kiswahili. Ce point est fondamental : le kiswahili est en train de développer, à mesure que se crée une littérature de prose écrite, une codification du style indirect. A la juxtaposition se substitue une subordination, qui affecte les marques verbales de la racine. Ainsi, les lecteurs comprennent que le discours est rapporté du point de vue de quelqu'un. Ce quelqu'un peut avoir des interlocuteurs: alors se crée la possibilité d'intégrer, parfois de façon implicite, une multiplicité de points de vue. Nous avons par cela donné un contenu à la notion de dialogisme élaborée par Mikhail Bakhtine et à cette polyphonie qu'il juge typique du roman. De fait un roman est un discours dans lequel sont reçus les discours multiples des acteurs sociaux, dans lequel ils entrent en dialogue, dans une construction qui nous demande d'accepter cette vision dialogique, donc politique et religieuse, suivant les acteurs dont les discours sont repris. Au monologisme, propre par exemple à la poésie épique ou aux odes guerrières, le roman substitue une structure polydiscursive et polyglossique que le discours de l'auteur narrateur, souvent omniscient, parfois personnage lui-même, produit. Le roman a donc partie liée avec notre modernité du fait de cette facilité, qui est une construction philologique, à laisser des points de 
vue multiples s'exprimer, à donner une expression à une réalité sociale, donc verbale, que le sujet seul ne peut jamais maîtriser même s'il peut en donner quelques aperçus.

En Europe même, le grand siècle de la philologie, à partir de la seconde partie du XIXe siècle, a vu l'affirmation de nombreuses langues littéraires (écrites), qu'il s'agisse du hongrois, du tchèque, ou du catalan. Cet âge d'or de la philologie a été aussi accompagné par la réflexion pragoise sur les langues littéraires et leurs fonctions:

Le Cercle de Prague ne propose pas uniquement une description réaliste de la langue littéraire. Ses membres, surtout Havránek et Mathesius, établissent une base théorique visant à situer la langue littéraire à l'intérieur des phénomènes linguistiques. Bien que cette théorie soit beaucoup moins connue dans le monde linguistique que la phonologie de l'École de Prague, elle constitue un des apports les plus importants de l'École en ce qui concerne notre compréhension de la langue. (Garvin, in Bédard et Maurais, 1983 : 145).

Ces travaux sont contemporains de l'œuvre de Bakhtine et éclairent son point de vue historicophilologique. Il traite de problèmes qu'il rencontre dans son activité professionnelle, sous le régime stalinien et dont il voit les implications politiques : le dialogisme et la polyphonie sont évidemment des notions anti-totalitaires; la novlang orwelienne ne leur fait aucune place. Mais les notions bakhtiniennes trouvent des points d'application particulièrement pertinents dans les langues de l'Afrique, écrites depuis la fin du XIXe siècle, encadrées par les églises, des académies ou des language boards dans le monde colonial. La liberté dialogique de la prose s'accommode mal du contexte tatillon de comités de censure moraux ou politiques. Si les romans africains en langues européennes sont publiés en Europe, ce n'est pas par hasard : on a souvent dit que leur public était en Europe, et c'est juste. L'étude du contrôle politique de la presse à la colonie est édifiante : la liberté d'expression n'existe pas. Dans le monde colonial africain, au moins jusqu'en 1960, s'exprimer dans une langue africaine, écrire un roman, est à la fois difficile et dangereux. Composer un récit qui mêle discours biblique, expression lyrique, slogans politiques parfois, calques vernaculaires, souvenirs de la tradition, provoque des effets de lecture surprenants chez des lecteurs qui ne connaissent souvent de l'écrit que ce qu'ils entendent à l'école biblique. Telle était la situation durant la première moitié du siècle, telle a été la situation du temps de l'apartheid. Tel est l'effet qu'ont provoqué les premiers textes de prose, de fiction en xhosa, en sesotho, en yorouba, ou en akan. Mais cet effet a rarement été analysé en détail. Ces romans ont été trop vite la proie de linguistes coloniaux peu habitués à l'analyse littéraire, peu sensibles à l'innovation artistique, et peu désireux de dégager les implications critiques, voire 
révolutionnaires, de certains de ces textes. C'est ce que je voudrais montrer par la suite à propos des romans de Thomas Mofolo et de Aniceti Kitereza.

Mais l'histoire de l'expression romanesque en langues africaines n'est pas finie et je voudrais aussi m'intéresser à la poursuite actuelle de ces pratiques d'écriture. Les langues africaines sont toujours parlées, et il se trouve des auteurs pour les écrire. L'effet roman n'a pas encore produit toutes ses conséquences et il est peut être souhaitable qu'il continue à se manifester dans de nouvelles langues si nous prenons le point de vue de la défense de la diversité culturelle, qui me semble être à l'origine de la «littérature comparée». Nous nous proposons donc une relecture des premiers textes dans chaque langue considérée dans notre séminaire, pour en dégager la charge d'innovation, perdue sous la gangue des commentaires et l'enduit des mauvaises traductions, souvent pire que l'absence de traduction : lecture sans complaisance des nouveaux textes, dans lesquels il faut faire le départ entre démarche novatrice, exprimant un rapport vivant et original à la langue, ou démarcage parfois appliqué de textes traduits. La littérature comparée, discipline à laquelle se rattache aujourd'hui le grand auteur kenyan, vivant en Californie, Ngugi Wa Thiongo, est alors en raison de sa tradition le lieu d'un tel débat, trop évité en France au profit des multiples variations d'une Francophonie évidemment unilingue, malgré qu'elle en ait et quoiqu'elle veuille nous faire accroire. Les soi-disant lectures polyphoniques de textes francophones sont des exercices de virtuosité interprétative, mais ne peuvent dissimuler la violence faite aux autres, que l'on a d'abord fait taire ou considérés comme muets.

\section{DES OBJETS RARES}

Il est évident que la méconnaissance des langues est un obstacle à la généralisation : rare qui peut prétendre lire dans plus de quatre ou cinq langues africaines. J'en ai étudié trois, et je peux en lire une. Je peux aussi juger à partir de cette dernière, le kiswahili, prototype des langues bantoues, de la structure de nombre de récits dans les autres langues bantoues. C'est d'ailleurs dans ces langues que, pour des raisons complexes, au premier rang desquelles doit aussi figurer l'apartheid, il existe souvent le plus de livres.

Seulement il me paraît important de faire commencer une réflexion comparatiste sur des données empiriques, sur une expérience pratique de la lecture, de l'effet que les livres, sous leur 
aspect le plus matériel peuvent faire. Ainsi, on peut s'interroger sur les raisons qui font qu'un ouvrage diffusé en feuilleton ne sort pas en volume. Plusieurs romans publiés dans des langues de l'Afrique et cités dans l'ouvrage que nous avons publié sont dans ce cas : en malgache, en xhosa, en fon. L'effet roman n'existe pas, dilué dans l'effet journal. De fait, le succès du roman sotho sous forme de feuilleton a tout de suite été suivi du succès sous forme de livre ; le livre a été connu sous son aspect matériel, réédité. Ce n'est pas le cas de tous les livres: beaucoup demeurent confidentiels, comme des brochures d'Organisations non gouvernementales, et ne circulent jamais comme de vrais livres. Ainsi, le «livre » xhosa est connu de quelques spécialistes, cités par Jeff Opland, du fait de son auteur, Samuel Mqayi, devenu une personnalité importante, mais personne n'a pu en produire un exemplaire jusqu'à aujourd'hui. La même situation prévalait au Mali où beaucoup avaient entendu parler d'un livre en bambara que personne n'avait vu.

Beaucoup de raisons viennent à l'esprit pour expliquer minceur et rareté des livres écrits en langues africaines : situation économique difficile, absence de lectorat solvable, mais aussi peut-être des raisons qui tiennent à l'imprimerie elle-même. Beaucoup de langues sont difficiles à lire, y compris par leurs locuteurs natifs et par les linguistes. Lire une langue tonale comme l'ewe ou le yoruba - j'ai beaucoup essayé - est pour un non-locuteur natif très difficile en l'absence des tons que les imprimeurs rechignent à noter. La moitié ou presque de l'information phonique est perdue dans une graphie trop réductrice. Cette situation émeut rarement les locuteurs qui semblent trop souvent persuadés que personne ne se lancera dans la lecture si complexe de ces textes.

Cette question a été ravivée au cours de notre séminaire par une double observation : en kiswahili, le roman d'Aniceti Kitereza est énorme. Il compte près de 600 pages, réparties en deux volumes de plus de deux millions de signes. La même remarque vaut pour le grand texte de Ngugi Wa Thiongo qui vient de sortir en traduction après avoir été publié en trois volumes indépendants, sortis l'un après l'autre (Murogi ya Kagogo). L'éditeur de Ngugi qui est aussi l'un de ses plus anciens soutiens, présente le titre comme « le plus gros livre paru dans une langue africaine, après la Bible ». Faire un gros livre, mais en anglais, était déjà l'objectif d'un concurrent de Ngugi, le romancier populaire David Maillu, auteur d'un «gros» livre: «greatest » African novel. La remarque de l'éditeur de Ngugi vient directement répondre au défi de Kitereza et aux six cents pages en kiswahili, traduites de la version originale de son roman. 
Affirmer une littérature vivante, c'est produire des romans, et le roman demande un certain volume. C'est ainsi que j'interprète le souci de Ngugi.

Lisant le cours de Roland Barthes sur la préparation du roman, j'ai noté que la question de la longueur l'intéressait, puisqu'il part de la notation minimale, celle du haiku; le roman, à l'inverse, étant en quelque sorte la possibilité de la notation maximale, voire interminable. Une telle physique du roman correspond à une pratique de la lecture. Il faut un texte lisible longtemps et facilement pendant longtemps, il faut une certaine fluidité, même si elle est méandreuse, mais il faut un flot. La parole est visqueuse, elle accroche, bute. L'écriture est ce flot, alors que la parole est une sorte de glu. Pour tenir longtemps la plume, il faut un instrument souple et fiable, en somme une langue littéraire. La montée de ce flot de paroles est la création de cette langue littéraire. La réflexion sur les langues littéraires a été menée avec clarté par des linguistes pragois et elle m'a beaucoup guidé dans mes recherches (Vachek 1964).

Comme je l'ai noté, on peut être un bon linguiste et ne pas « savoir» lire une langue que l'on parle, ou plutôt ne jamais la lire. Le roman est le vecteur de l'innovation littéraire, parce qu'il permet au flot de couler sans entraves. Au scripteur de donner son rythme, d'imprimer sa marque. Les notations de Ngugi sont tout à fait éclairantes pour notre propos :

Le gikuyu n'a pas de tradition d'écriture de fiction, ou d'écriture romanesque. Gakaara a essayé de créer une telle tradition mais ses livres furent interdits dans les années cinquante. Il créa alors un journal appelé Guikuyu na Mumbi dans lequel il y avait un feuilleton appelé Kiwai na Nduna. Mais ces textes n'avaient pas la qualité de ses écrits des années cinquante qui n'étaient plus disponibles. Je fus aussi confronté aux questions de mots, de temps, et même avec la question de l'impression visuelle produite par les mots sur le papier.

Les mots et les temps sont d'autant plus fuyants que l'orthographe gikuyu est peu satisfaisante. La langue a été réduite à l'écriture par des non-locuteurs natifs qui ne pouvaient pas bien identifier les longueurs des voyelles. La distinction entre voyelles longues et brèves est très importante dans la prose et la poésie gikuyu. Mais la graphie actuelle laisse souvent le lecteur deviner s'il s'agit d'une longue ou d'une brève. Cela devient très fatigant dans un morceau important de prose. De plus ce manque de distinction assume en fait une connaissance préalable de la langue de la part du lecteur! J'ai essayé de tenir compte du problème en écrivant deux voyelles là où il y avait des longues. Mais cela m'a pris plusieurs pages, avant que je m'habitue à ce procédé. Et ce procédé ne m'a pas totalement satisfait car ce qu'il faut c'est une nouvelle lettre ou un nouveau signe diacritique pour la voyelle longue. Le gikuyu est aussi une langue à tons mais la graphie n'indique pas les variations tonales! (Ngugi Wa Thiongo, $1986: 74$ )

L'insatisfaction du romancier porte d'abord sur l'instrument, sur cette langue littéraire qui n'a pas la fluidité requise. Mais notre auteur a du métier, de la constance, il s'acharne et il réussit à donner de gros livres : il sait que se joue dans cette capacité à fournir du texte une partie dont l'enjeu n'est pas suelement quantitatif. 
Nous avons donc étudié la situation dans vingt-sept langues et publié des essais sur vingt langues. Nous avons cherché à être pratiques, en donnant la priorité à la circulation de ces objets littéraires, mais les textes peuvent se regrouper en diverses catégories.

Je voudrais ici m'intéresser aux marges de notre entreprise, aux premiers textes et aux derniers. Je me demande comment promouvoir les petites langues ? contribuer à la biodiversité culturelle? avoir un régime de lecture équilibré ? Ces vastes questions suggèrent des réponses générales, qui portent par exemple sur la traduction des œuvres, sur leur édition et leur interprétation, qui permettent de les inclure dans des programmes scolaires et universitaires. Ils demandent aussi une attitude critique et militante face aux grosses machines du mondoromanzo, les bestsellers multinationaux (comme ceux de Paolo Coelho, Danielle Steel) qui ne disent pas tout sur notre monde.

Intéressons nous d'abord à quelques-unes des langues qui ont récemment vu surgir le roman dans leur production littéraire. Demandons nous ensuite comment ce surgissement a été compris et lu quand il s'est produit, il y a longtemps, dans d'autres langues de l'Afrique. Il est clair que l'Afrique pose, de manière aiguë, la question des langues de création. Cela est bien connu, mais il faut entrer dans les détails.

\section{QUELQUES EXPERIENCES LIMITES}

L'écriture du roman est un pas décisif dans la littérarisation d'une langue. J'ai insisté sur la circulation des textes : il importe qu'ils existent en volume pour produire un effet, que l'objet roman ait une présence matérielle, que l'on n'en reste pas au journal ou à la mince brochure. Toute langue écrite a produit des romans. Tel est du moins ce que disent les locuteurs informés de la question. Et s'il n'y a pas encore de romans, dans certains cas, nos interlocuteurs s'attribuent le mérite de la naissance du genre dans leur langue. C'est ainsi que notre collègue spécialiste du sango me confia avoir écrit un roman, alors qu'un autre collègue me signala qu'il était le premier romancier en fon, ce qui lui vaut de figurer dans notre ouvrage. Ces découvertes sont un peu surprenantes, mais symptomatiques. Il y a une forme de dignité culturelle pour une littérature à produire des romans, une langue qui s'écrit doit pouvoir produire de longs textes en

prose. Par exemple la foire de Bruxelles en 1952 attribue un prix à un texte en français et un autre 
à un roman en kinyarwanda. Ce roman est-il publié ? Sans doute, mais nous n'avons pu en voir un exemplaire ni même en obtenir une description physique complète.

Ces textes demeurent trop souvent confidentiels. Certains restent en manuscrits, d'autres, reproduits à quelques exemplaires sous forme de brochure, ne sont pas vraiment des romans $\mathrm{du}$ point de vue du lecteur. Pourtant, un phénomène nouveau s'est produit après le travail de Ngugi Wa Thiongo : des romanciers ou des écrivains confirmés, connus pour leur travail en français ou en anglais, ont publié dans leur langue. Pius Ngandu Nkashama a écrit plusieurs romans en ciluba (cités in Tshisungu, 2006), Boubacar Boris Diop a écrit en wolof. On peut s'interroger sur l'effet de cette pratique en l'absence de traductions, voire de commentaires ou d'édition critique. Comment situer ces textes dans l'espace synchronique de la communauté des locuteurs et dans la diachronie du développement de la langue ? Dans le cas du ciluba, il semble que le romancier francophone confirmé, Pius Ngandu Nkashama, soit le premier romancier-auteur de deux textes publiés en ciluba, dont l'un est publié à Lubumbashi, mais qu'il n'ait fait que peu d'émules (Tshisungu, 2006). On est donc en face d'une pratique littéraire expérimentale dont l'écho dans la communauté linguistique semble réduit, en l'absence d'autres partenaires. Notons cependant que le texte de Ngandu est publié au Congo (ex Zaïre) et qu'il traite des moments dramatiques de la répression mobutiste.

Dans bien des cas, l'historiographie laisse à désirer, en particulier dans le cas des langues de l'ancien Zaïre, malgré les travaux récents sur le ciluba. Ainsi, dans le cas du kikongo, langue dont la longue histoire avec écriture devrait mériter l'intérêt des historiens, il est difficile de réfléchir sur le roman. Une adaptation de Robinson Crusoe (Nsamu wa Nsau Kuloso, 1928) est souvent citée comme premier roman (Mbelolo, 1972 : 134). Le texte d'un missionnaire suédois J. Petterson, Nsamu a Mpanzu 1 (Vie de Mpanzu, 1935) en trois volumes $(2,3,1938)$ est « le plus long roman qui ait jamais été écrit en kikongo » (Mbelolo, 1972 : 136). L'article de Mbelolo pourrait constituer le modèle de ce type de monographie: il donne aussi le nom d'Emile Dinsengomoka, dont le livre Kwenkwenda, paru en 1943, aurait été un "vrai succès d'écrivain » (Mbelolo, 1972 : 139). La grande carence est celle de l'historiographie : peu de textes sont parus, rares sont les synthèses, et les informations bibliographiques laissent souvent à désirer. Il est difficile d'avoir, par exemple sur le lingala, une notion précise de l'état de la production de fiction. 
Les régions dans lesquelles la pratique écrite des langues africaines est la plus réduite sont en général celles des anciennes colonies françaises, notamment de l'Afrique de l'ouest. Or il existe une pratique littéraire romanesque en langues africaines dans ces régions aujourd'hui, mais elle pose de nombreuses questions. Le cas du roman peul est à cet égard fort différent des cas wolof, bambara, moore et fon. On connaît l'importance de cette langue, et de ses nombreux dialectes, parlée du Fouta Toro sénégalais à l'Adamawa camerounais, sur une vaste aire du Sahel. Elle est aussi écrite depuis longtemps en caractères arabes, ce qui n'est en rien une garantie de lisibilité générale. Amadou Hampâte Bâ a expliqué les problèmes liés à la graphie du peul. Il a aussi contribué à créer une graphie standard - adoptée à la Conférence internationale de Bamako en 1966 - donc à préparer l'instrument dans lequel pourrait se couler un nouveau discours plus fluide, plus indépendant des contraintes des traditions graphiques anciennes. Il a fallu un peu plus de dix ans pour qu'apparaisse, en peul le premier roman, Ndikkiri joom moolo, de Yero Dooro Jallo (1981). Sa parution coïncide aussi avec la mise à disposition d'un instrument graphique nouveau.

Ndikkiri joom moolo, le grand Dikko à la petite guitare, ou Ndikkiri, le guitariste, fiction relativement longue, mettant en scène des personnages imaginaires [...] dans laquelle le personnage principal s'illustre par les écarts de son comportement vis-à-vis des normes socioculturelles, qu'il s'agisse de ses mauvais tours ou de ses satires, qui ridiculisent les personnages les plus respectés de la société, particulièrement les marabouts et les notables (Aliou Mohamadou, in Garnier / Ricard, 2006 : 218).

Aliou Mohammadou étudie la graphie utilisée dans le texte dit du Caire. Elle est en rupture avec la graphie ajami et constitue une innovation, indépendante du travail de la conférence de Bamako. Cette innovation est liée à une rupture politique : les étudiants du Caire sont anticolonialistes et se situent en dehors de l'orbite ouest-africaine «néocoloniale ». Ils offrent une nouvelle vision du monde, ils ont un nouvel instrument, et le roman s'inscrit à point nommé dans ce contexte de rupture avec l'ordre ancien, qu'il s'agisse de l'ordre colonial, ou des diverses affiliations musulmanes. Le héros de Yero Jallo, «premier anti-héros peul » (FagerbergDiallo, 1995), est un individu qui fait éclater les cadres, et qui fait aussi éclater les graphies. Ndikkiri déchire, Allah recoud, Ndikkiri est « celui dont les actes les plus récents empêchent de parler des plus anciens » (Yero Dooro Jallo, Exergue de la première édition, 1981). C'est une sorte de trickster, un personnage picaresque : le genre romanesque en peul coïncide avec un moment de première rupture des cadres politiques et religieux. 
Le succès vient, l'écho est fort et le texte est alors rendu dans la graphie de Bamako, en 1993, graphie qui va être enseignée et devenir officielle, quand la langue a un statut.

Il apparaît donc que, si la première édition de l'ouvrage date de 1981, celui-ci n'a connu sa forme définitive qu'en 1994. Les différentes réécritures du texte résument à la fois le cheminement de l'auteur et les étapes que le peul est en train de parcourir dans sa modernisation: choix d'un système d'écriture, choix orthographiques, choix d'un standard commun, cette dernière question étant progressivement abandonnée, tandis que l'écriture de la langue évolue de plus en plus vers une latinisation en se référant aux recommandations de la conférence de Bamako de 1966.

Ces tâtonnements montrent également que le pas vers la modernité, en ce qui concerne du moins le peul et tout particulièrement le Sénégal et la Mauritanie, reste avant tout le fait des militants dans un mouvement qui doit beaucoup aux initiatives des étudiants de langue peule dans les pays arabes qui sont rejoints ensuite par de nombreuses associations en Afrique et en Europe. (Mohammadou, in Garnier / Ricard, 2006 : 217)

L'exemple peul nous paraît tout à fait significatif par la coïncidence entre la naissance du genre, l'affirmation d'une graphie, une crise politique et la création d'un personnage inclassable et dérangeant. Le roman est bien là pour permettre de dire ce qui ne peut se dire ailleurs, ce qui n'a pas d'autre lieu et qui en même temps est adressé à la communauté linguistique, qu'il invite à avancer. Le roman est aussi un vecteur. Yero Dooro Jallo anime des associations pour l'écriture en peul, il y a eu d'autres textes, une culture graphique apparaît peu à peu au point de rencontre de toutes ces tensions.

Le cas du bambara est analysé par Jean Derive (in Garnier / Ricard, 2006) dans un article sur le premier roman bambara, Kanuya Wale (Un acte d'amour, 1996). Notons d'abord l'éclatement dialectal de la langue, qui est enseignée en France à l'Institut national des langues orientales. Elle a été l'une des premières langues africaines étudiées en France, mais une certaine ambiguïté subsiste sur les dénominations (bambara, dyula, bamana, malinke). Cette langue est largement parlée au Mali et écrite dans le cadre des campagnes d'alphabétisation depuis plusieurs décennies. Une importante littérature orale a été transcrite et traduite. Dans ces campagnes d'alphabétisation, de nombreuses brochures, des pièces de théâtre, des essais, des journaux ont été publiés, mais il est difficile de citer d'autres publications écrites, de fiction ou de poésie. La recherche d'un long texte en prose a été une quête difficile auprès des spécialistes et en particulier auprès des spécialistes de l'alphabétisation. L'effet roman n'était pas véritablement présent. Pourtant un long texte, paru à Bamako, existe, que Jean Derive analyse en détail. Pour lui l'histoire des aventures du héros de ce texte, un chauffeur, donne lieu à un «roman didactique, dont l'histoire est un prétexte permettant à l'auteur de donner son point de vue sur certains 
problèmes de société afin de parfaire l'éducation morale et civique de ses lecteurs » (Derive, in Garnier / Ricard : 265-285).

De plus, ce texte est écrit dans une langue particulière, différente du bambara couramment employé à l'oral. Jean Derive cite la réflexion d'un locuteur : «C'est comme si c'était du bambara traduit d'une langue étrangère. » (Derive, ibid. : 279) Or c'est bien de cela qu'il s'agit, comme il le montre en poursuivant son analyse. L'auteur a reçu commande d'une organisation de développement canadienne, écrit son texte en français et l'a traduit lui-même en bambara. Jean Derive a pu consulter le tapuscrit français produit en 1996. Il est donc compréhensible que ressorte, du point de vue du locuteur urbain contemporain, l'impression d'un certain artificialisme des traductions, une rigidité qui n'est pas celle du parler quotidien, d'autant plus que la première version en français est marquée par une conception assez scolaire de la littérature, du beau style et d'une idée conformiste de la « belle langue » : floraison d'adjectifs, emphase, longues phrases qui passent difficilement en bambara.

Dans le cas du bambara, la langue est enseignée aux adultes, largement parlée, et il faut des textes. Le livre fait donc partie d'une opération de développement de l'alphabétisation, associée à une éducation des jeunes et des adultes sur les problèmes liés au SIDA.

Cette situation malienne se retrouve en partie au Burkina Faso, où malgré les discours officiels sur la défense des langues de l'Afrique, le français semble la seule langue pratiquée par les écrivains. Le best-seller moore est un recueil d'histoires drôles, La-y zem zem (Riez doucement) d'Halibou Ouedraogo, réédité, et soutenu par une agence de coopération (Sissao, 2005). Il y a là un exemple qui mériterait d'être retenu et étudié en tant que tel. Ce texte considéré comme fondateur et qui ne semble pas avoir eu de postérité, n'a suscité que peu d'analyses. Dans un entretien demeuré inédit (Sissao, 2003), l'auteur déclare poursuivre une œuvre basée sur « les conseils, la morale, par le biais de l'humour »; il note aussi le peu de formation littéraire des écrivains burkinabe en langues nationales. Il en tire la conclusion que l'amélioration de la qualité de leurs manuscrits et leur production passe par une formation aux « règles de transcription » des langues nationales, ce qui ne me paraît pas évident, à moins qu'il ne s'agisse de simplifications, qui seraient bien utiles au non-spécialiste dans le cas de la graphie du moore. Le chiffre de plusieurs tirages de 10000 exemplaires est avancé, après une première édition en 1997. Nous sommes alors surpris de voir que ce type d'ouvrage est laissé de côté par les défenseurs des langues nationales qui ne semblent pas soucieux d'analyser sa diffusion, les raisons et les limites 
de son succès : il ne se présente pas avec les garanties de légitimité intellectuelle, l'auteur n'est qu'agent d'alphabétisation que l'étiquette de « roman » lui semble - bien à tort - conférer.

$\mathrm{Au}$ terme de notre séminaire, nous n'avons pu avoir un point de vue clair sur la production littéraire en wolof. Certes, une abondante littérature en wolof existe, impulsée par des militants comme Pathé Diagne, auteur d'essais et de travaux scientifiques. La vie intellectuelle sénégalaise s'est nourrie des conflits entre le président Senghor et des intellectuels qui, comme Sembène Ousmane, voulaient rendre leurs œuvres accessibles dans la langue de la rue. Sembène est finalement devenu cinéaste, ses films sont parlés en wolof et sous-titrés en français, et le roman en wolof est resté un objet universitaire. Pourtant, d'autres écrivains n'ont pas renoncé. Cheikh Ndao a toujours voulu créer en wolof, mais son œuvre est publiée uniquement par les éditions de l'IFAN. Il en est de même pour le roman de Mame Younouss Dieng, qui serait l'auteure du premier roman, Aawo Bi (Sarr, in Garnier / Ricard, 2006).

Cheikh Ndao a certes écrit des nouvelles de quelques dizaines de pages en wolof. Il aurait aussi écrit son premier roman, Buur Tillen, en 1967, mais n'aurait pas réussi à trouver d'éditeur, si bien qu'il fut contraint d'adapter (non pas de traduire) son roman en français, avant de le publier en version abrégée en wolof (Sarr, in Garnier /Ricard, 2006 : 311). Est-ce assez pour créer l'effet roman? Je ne le crois pas. Cette confusion me semble symptomatique : les écrivains wolof sont des écrivains bilingues, leur entreprise est une traduction, une démonstration, une véritable expérience littéraire qui demande à être analysée en tant que telle. Il semble y avoir un va et vient entre les langues, qui témoigne d'une volonté démonstrative plus que de la volonté de dire en wolof ce qui ne peut pas être dit autrement.

Les textes de fiction recensés ne dépassent pas les 75 pages, comme le roman de Mame Younouss Dieng (Maam Yunus JED, dans la graphie wolofe) Aawo Bi (Dakar, 1992, 72 p.). Ce livre est, comme celui de Cheikh Ndao, publié par l'IFAN, donc toujours inscrit dans un cadre officiel. Ces textes, édités par des institutions dans le cadre de programmes de recherche, ne relèvent pas de l'édition commerciale. Cela ne semble pas être le cas de Doomi Golo qui présente une véritable rupture. Le propos de Boubacar Boris Diop, avec ce roman, est bien de déplacer le débat et de jouer sur l'effet que ne peut manquer de provoquer un roman, un objet roman, arraché au domaine de l'expérience linguistique. L'auteur explique cette rupture par le traumatisme provoqué chez lui par l'attitude française face au génocide rwandais. Il aurait aussi eu besoin de prendre des distances par rapport à une francophonie politiquement irresponsable. C'est là une 
attitude compréhensible et intéressante même si elle ne peut résumer l'ensemble de la pratique littéraire de Boubacar Boris Diop, qui ne souhaite pas en rester à cet arrachement.

La littérature en peul comme la littérature en wolof et en bambara sont récentes. Alors que la littérature en peul est le produit d'une expérience personnelle originale, la littérature en bambara est, semble-t-il, le fruit d'une commande d'ONG. En wolof, le cas est plus complexe : il semble y avoir le souci de démontrer que la création romanesque est possible. Cela fut fait, mais à peu de frais, et au prix de grandes difficultés rapportées par les commentateurs avec une certaine complaisance. C'est contre cette démonstration «à l'économie » - j'ai du mal à croire que Cheikh Ndao ne puisse trouver un éditeur pour son roman en wolof - que réagit Boubacar Boris Diop avec son roman de trois cents pages.

Le cas du fon peut être compris dans cette même perspective. Jean Norbert Vignonde, auteur du premier roman en fon, Bo Gbe Die A (Quelle vie !, Paris, 1980), retrace les étapes de sa propre transformation en romancier. L'auteur est un critique et un historien de la littérature, auteur de nombreux articles en français sur les littératures de la côte du Bénin. La révolution béninoise, en 1972, a beaucoup insisté sur l'utilisation des langues africaines et il s'est associé à cette revendication. Il a voulu développer le corpus littéraire en fon qui ne comprenait pas la fiction. Son travail est donc le fruit d'un volontarisme politique, un peu analogue à celui qui anime Ngugi. Pour l'auteur il s'agit bien d'une expérience, qu'il appelle une «étude de faisabilité ». Seulement, ce projet a des difficultés à trouver des lecteurs, à émerger dans la communication publique. Remarquons que la transcription du fon est particulièrement complexe, et tout à fait différente de celle du yorouba voisin, ce qui a peut être une forme d'effet dissuasif auprès des futurs lecteurs. Le texte est demeuré un feuilleton et l'auteur nous annonce dans sa communication à notre ouvrage que le livre va paraître en volume, près d'un quart de siècle après sa rédaction. Sa nécessité est pédagogique et politique, comme le texte en bambara. La difficulté est de l'articuler avec le mouvement propre de la langue et de la société. De plus, ce qui nous est raconté plonge au cœur des problèmes politiques. Le héros d'un premier roman, d'un roman émergent, peut-il ignorer qu'il fait œuvre de pionnier ? La révolution qui a été le déclencheur de cette envie d'écrire est peut être aussi ce qui empêche le texte de se diffuser : les problématiques bakhtiniennes sur le dialogisme et le totalitarisme peuvent ici être évoquées. Un régime marxoïde comme celui de Kerekou au Bénin (de 1972 à 1989) n’encourageait pas le débat, ni la critique. Il 
y a donc là une contradiction profonde, qui demande à être problématisée, mais il est clair qu'il est plus facile d'exprimer cette exigence que de s'y soumettre.

Ces questions sont aussi celles que pose le travail de Ngugi, considéré dans son développement historique. Ce qui a commencé au Kenya, à Limuru, dans un centre social, ce qui a continué en résidence forcée, puis en exil, ce qui a été une rupture est aujourd'hui une entreprise culturelle mondiale, menée depuis la Californie. De plus, cette action se veut un modèle pour d'autres communautés linguistiques «minoritaires", comme le propos actuel de Ngugi l'indique clairement.

\section{LES PIONNIERS N'ONT PAS ETE LUS}

La réflexion sur les premiers romans gagne à se compléter d'exemples historiques, et je trouve particulièrement intéressant le rapprochement avec le roman hellénistique Ethiopiques, premier roman occidental pour Pavel $^{2}$. Ce texte suscitait l'admiration de Cervantes qui en a donné une adaptation. L'idée fondamentale est qu'il y a une pensée du roman et que la simple compréhension de son propos suppose tout un arrière-plan, en quelque sorte institutionnel. A ce titre, le rapport entre le conte et le roman, trop souvent posé comme évident, devient hautement problématique, voire même incohérent pour Pavel. Sa démonstration qui vaut pour le rapport entre les contes et les premiers romans hellénistiques me paraît de nature à éclairer le thème rebattu du passage de l'oral à l'écrit qui omet trop les questions épistémologiques :

Pour vraisemblable qu'il puisse paraître, ce rapprochement avec l'une des formes narratives les plus archaïques n'est cependant qu'un leurre car [...] les héros du roman grec exhibent bien les rudiments d'une propriété nouvelle, l'intériorité [...] le véritable sens des aventures romanesques est la sauvegarde d'un espace intérieur, à peine désigné, lieu de l'amour, de la piété et du respect des normes. (Pavel, 2003 : 64-65)

Le personnage est doté d'une volonté de rupture : il est une victime, mais aussi un acteur de sa propre existence.

La découverte du caractère relativement aléatoire du sort humain est un résultat tardif et considérable de la pensée. Comprendre que l'action humaine et ses fruits ne sont pas motivés dans chacun de leurs détails implique une cosmologie qui s'est libérée du besoin primitif - incarné dans la mythologie polythéiste - de joindre à tout événement des justifications qui soient locales et cosmiques (Pavel, 2003 : 67).

\footnotetext{
${ }^{2}$ Ethiopiques, traduites en français en 1547, en espagnol en 1554, en allemand en 1554, en italien en 1556, en anglais
} en 1567. 
Dans l'étude des genres de la littérature écrite en langues africaines, nous nous trouvons devant de difficiles problèmes théoriques : le premier concerne la place de la prose dans une langue littéraire en formation, et il relève de l'histoire et de la philologie. Ces textes, ces romans, sont souvent produits dans des Missions, au contact d'un monde dans lequel théologie, politique, éthique sont intimement mêlés, ce que nous avons du mal à comprendre aujourd'hui.

Les questions du christianisme, liées à la personne, à la liberté, au choix du destin, s'expriment dans les premiers romans, souvent publiés dans des presses missionnaires, comme Moeti Oa Bochabela de Thomas Mofolo. L'action humaine, prise entre le caractère aléatoire du sort humain et la reconnaissance de l'espace intérieur de la délibération éthique, voilà qui est peut être le lieu propre du roman. La quête du sens dans un monde où règne la violence est d'abord une quête individuelle : le groupe ne donnera pas la réponse, même s'il garantit des formes de solidarité, voire d'insertion, par la poésie par exemple. Il faut partir seul : le chemin ne peut se faire qu'individuellement. Mofolo, en posant cette exigence, est profondément chrétien : la liberté individuelle est le premier bien de l'homme : à lui de faire ses choix, et non à la tribu, au clan ou à la classe d'âge. A un certain moment, il faut savoir rompre avec ses anciennes solidarités : Jésus a eu des mots très clairs sur ce point (Evangile selon St. Matthieu, 10, 37). Quête du sens, espace intérieur de la délibération, voilà sans doute de bonnes raisons pour que le texte écrit soit fluide. Le Mosotho qui devient chrétien doit-il changer de monde? Mofolo explore certaines des voies de cette conversion. Je me permets de suivre ici l'analyse que nous donnons de cette situation (Garnier / Ricard, 2006 : 30-32).

Sa quête amène Thomas Mofolo à réfléchir sur les traditions rapportées par son peuple et notamment sur le mythe d'origine des hommes, dont il nous donne une version dans son texte. Elle l'amène à inventer des mondes et à voyager dans une géographie mystique. Il écrit un sesotho qui n'a jamais été parlé, ni même écrit ainsi, comme le montre Daniel Kunene. Cette nouvelle langue, au service d'une vision du monde entièrement originale, est portée par une réflexion sur la liberté. Tout cela fait de Moeti oa Bochabela $(1907,2003)$ un roman, c'est-à-dire une œuvre de fiction en prose qui ne ressemble à rien de connu en sesotho, « un ouvrage d'imagination absolument original» (Livre d'Or de la Mission du Lesotho, 1912, p. 507) : ce n'est pas un conte, ce n'est pas un récit comme ceux de Motsamai, tirés des souvenirs de locuteurs aventureux comme Au temps des cannibales (1999, 1ère édition : 1912), ce n'est pas un 
poème d'éloges : c'est une interrogation, un questionnement sur le sens de la vie, une fiction en prose qui porte des interrogations éthiques sous la forme d'un récit. Ces interrogations font éclater la société villageoise ancienne, mais en fait elles mettent profondément en question n'importe quelle société, car la mystique ne fait pas bon ménage avec la politique.

Le début du roman annonce ses thèmes, mais il faut que ces questions trouvent le moyen de se formuler. C'est ici que l'histoire de la marche ou de la démarche de Fékisi est neuve chez les Basotho. Le plus grand héros des Basotho, le roi Moshesh, celui qui a refusé de dévorer son peuple, a aussi refusé d'être baptisé peu de temps avant la naissance de Mofolo, en 1870. C'était un homme juste et il a le respect de tout son peuple : seulement le monde change et le sens n'est plus dans ce que les ancêtres ont transmis. Les Blancs sont arrivés, ont pris une partie du pouvoir, voire tout le pouvoir en Afrique du sud. L'homme africain se trouve sommé de réagir : quel sens tout cela a-t-il pour lui ? Le roman donne sens à une expérience neuve du monde et il s'inscrit bien dans l'histoire d'un genre dont il utilise pour la première fois en Afrique le potentiel original.

Thomas Mofolo est un écrivain présent dans le monde de son époque. Il est né à Khojoane, près de Mafeteng, sur la frontière avec l'Afrique du sud. Mofolo fut toute sa vie actif sur la frontière entre le Lesotho et l'Afrique du sud et il a été l'élève des écoles de la Mission de Paris dans cette région. Il travailla en Afrique du sud, créant une agence de recrutement pour les mines. Dans une thèse récente, il est question de son petit empire commercial (small empire) (Maloka, 1995 : 95), ce qui permet de mesurer à quel point il avait réussi à créer une entreprise rentable. A Teyateyaneng, sur la frontière avec l'Afrique du sud, le principal centre commercial de la grand rue est le centre Mofolo, qui appartient encore aujourd'hui à sa famille. Dans le roman Pitseng, il nous raconte comment les élèves du Lesotho vont dans les écoles d'Afrique du sud, il rend compte des échanges entre les Xhosa, les Bathepou, et les Basotho. Son roman Chaka est un roman sur la région et l'Afrique et pas seulement sur le Lesotho. Aujourd'hui encore, les postes frontières, Tele Bridge par exemple, voient passer de nombreux élèves scolarisés de l'autre côté de la frontière, dans ce qui était naguère le bantoustan du Transkei. Enfin, Thomas Mofolo fut victime des lois sur la propriété terrienne, et perdit la ferme qu'il avait acquise à côté de Rhodes. Il s'agissait pour un historien contemporain, Edward Maloka (1995 : 95), d'un essai « futile » pour s'affranchir de lois très strictes qui limitaient les propriétés des Noirs en Afrique du sud. Cette région dans laquelle le Transkei xhosa, l'Afrique du sud blanche et le Lesotho se 
rencontrent, était celle dans laquelle Mofolo a grandi. Aliwal nord, fréquemment mentionné dans Pitseng, est une ville centrale dans la geste boer. Les Voortrekkers franchirent ici l'Orange, à gué, en 1836, pour entrer dans la Transorangia, ce qui devint le Free State. Le sud du Lesotho est donc excentré par rapport au siège du pouvoir royal, mais au contact direct de l'Afrique du sud industrielle ; Aliwal a une gare à partir de laquelle le contact est établi avec les ports du sud (East London, Port Elizabeth) et Kimberley. L'auteur d'un premier roman mystique était un homme profondément engagé dans la vie sociale de son pays et de son temps, et cela aussi aida à faire de lui un romancier.

L'écrivain devient parfois un prophète, il peut se comparer aux autres prophètes bantous de la même époque (Sundkler, 1948): tel est bien le cas de Mofolo qui entrevoit une africanisation du chritianisme dans son premier roman, Moeti oa Bochabela. Cette africanisation a trouvé, comme nous le savons, des traductions sociales réelles avec les Eglises africaines. Une force de l'utopie pénètre les textes de Mofolo et donne à son premier roman une tonalité particulière : il est à la fois très inséré dans la réalité sociale et en même temps, il voit un autre monde, celui d'une foi en mouvement qui, si elle ne déplace pas les montagnes, gravit la montagne de Sion - dans le dernier chapitre de Moeti oa bochabela.

Le premier grand roman swahili, Bwana Myombekere na Bibi Bugonoka (M et sa femme B) d'Aniceti Kitereza, n'est pas qu'une simple description romancée de la vie dans les îles Ukerewe, situées au milieu du Lac Victoria. Il est tout d'abord traduit d'une première version en kikerewe : cela est dit dans l'introduction, mais pas sur la page de titre. Il y a déjà dans cette création swahili virtuelle une forme d'utopie : l'auteur crée un monde imaginaire qui ne relève pas d'une démarche prophétique. Dans le monde de la mission catholique, l'initiative individuelle de l'homme de foi a moins de place; le prophétisme n'est pas une pratique acceptée. Comme chez Mofolo, ni la Mission ni le christianisme ne sont explicitement présents, alors que le livre peut se comprendre aussi comme un exercice de pratique des vertus, humaines et chrétiennes. Myombekere essaie de vivre une vie conjugale: il met à retrouver sa femme une sorte d'héroïsme admirable, qui situe leur aventure dans un cadre harmonieux, et très particulier, celui des îles Ukerewe. Le caractère de ces îles en fait des lieux de refuge, certes dominés par le clan Silanga, clan royal des Sese, alors que les Wasukuma, les Jita et les Kara participent à la culture de ces îles. Le nom de Bakerewe sera donné au début du XXe siècle. En d'autres termes, les traditions ancestrales du clan royal ont été soumises à la pression des immigrants et aux 
innovations apportées par les Pères blancs qui ont façonné au XXe siècle une partie de la culture des Lacs. Le but de l'auteur est bien d'inciter à être fiers des traditions communes des Bakerewe, mais il a, selon un anthropologue, spécialiste de ces îles, «brillamment évité de faire de cette histoire un récit 'sese' particulariste, qui aurait montré seulement son clan et sa chefferie sous un jour favorable. Il transcende la diversité ethnique et les particularités des îles, car il a choisi de mettre l'accent sur la famille individuelle, en insistant sur la sagesse pratique et les codes éthiques présents dans la société » (Hartwig, 1972 : 164, 168). Ce jugement, formulé par un anthropologue connaissant la culture kerebe me paraît tout à fait recevable à la lecture du texte. L'aventure du héros ne lui fait pas quitter la communauté villageoise comme dans Moeti; au contraire, elle contribue à la faire vivre encore mieux. Telle est bien la dimension utopique de ce texte.

Le roman est une rupture culturelle et une innovation radicale ; l'arrivée de l'écriture, de la monnaie, des colons, du christianisme chez les Bakerewe, provoque chez certains sujets la mise à distance du mythe ancien, face auquel se trouve la fiction utopique. Le mythe est mis en perspective, repensé, dramatisé : le chapitre 19 nous raconte le mythe fondateur des relations entre hommes et femmes, alors que les autres chapitres nous donnent la version romancée d'un nouveau projet de cohabitation, une nouvelle façon pratique de gérer ces relations à travers l'exemple des deux héros du livre. Une lecture ethnologique manquerait de traduire la nouveauté de cette histoire. Kitereza, en tant que romancier, regarde l'avenir, même s'il nous parle des traditions.

Créer un monde de rêve et le faire passer pour le monde réel, tel est le propre de l'utopie. Décrire les travaux et les jours d'un village africain en accordant l'homme à la nature, et la nature à Dieu, voilà bien qui est une sorte de rêve. Quand, de plus, ce Dieu n'a rien qui le distingue du Dieu des chrétiens et que le monde qui nous est décrit est un monde où les valeurs humaines et chrétiennes sont au centre de l'action, ne peut-on parler d'utopie chrétienne?

\section{LA NOUVELLE ECONOMIE DE LA LITTERATURE ET LA PLACE DU ROMAN}

Dans la nouvelle économie du roman qui se met en place, ces textes ont peu de chances d'être réédités. Nous avons identifié des productions romanesques dans 27 langues et ce chiffre n'est évidemment pas exhaustif. Pourtant, il dit quand même que la pratique dur roman est très 
limitée par rapport aux centaines de langues disposant d'une tradition écrite et par exemple d'une traduction de la Bible. Il n'y a que peu de romans : la fiction est une pratique textuelle innovatrice et complexe, pourtant elle est sans doute un passage obligé pour la survie des langues en tant que media de création originaux. Edouard Glissant l'a dit avec clarté et prescience, à Québec, en 1972, dans un texte repris dans le Discours antillais (1981) :

Un peuple qu'on réduirait à la seule pratique orale de sa langue serait aujourd'hui (et quoique nous pensions de l'illégitime d'une telle fatalité) un peuple voué à la mort culturelle, laquelle n'est jamais que le blême reflet d'une agonie autrement réelle. (Glissant, 1981 : 316)

Tout mon travail n'est en somme qu'un commentaire de son propos. L'économie actuelle des best-sellers romanesques au niveau mondial montre une progression, voire une domination des gros livres, souvent traduits de l'anglais: rares sont les titres traduits de petites langues. La mondialisation de la culture opère partout, même là où on l'attendrait le moins. Un film comme Mondovino, primé à Cannes en 2004, montre combien il se produit une homogénéisation du goût du vin pour répondre aux goûts de prescripteurs médiatiques. Des technologies viennent à la rescousse, des media célèbrent ces innovations. Et face à cette mondialisation de la fiction, à ce «mondoromanzo », des formes et des langues originales « minoritaires », voire subalternes, n'ont que peu de chances de se faire entendre. Nos vingt romans écrits dans des langues africaines semblent voués à demeurer dans les bibliothèques des érudits. On comprend alors que de jeunes écrivains, soucieux de se faire entendre, hésitent à s’exprimer dans leur propre langue.

Rares sont les romans contemporains qui ont réussi à trouver une place dans la lecture en Afrique : beaucoup sont des tours de force linguistique, ou des démonstrations de compétence linguistique et littéraire. Ce problème est au cœur de la démarche du centre que dirige Ngugi et je ne peux mieux faire que citer son argumentaire : il vise à «défendre le travail politique de la traduction, dans l'impératif double de préserver les textes, en particulier dans des langues moins connues et de disséminer ces œuvres largement grâce à l'anglais et à la traduction en d'autres langues ». On ne saurait mieux dire, on ne saurait être plus clair pour favoriser cette conversation entre les langues, les cultures et les disciplines, qui est à la base de la communauté multiculturelle naissante. Suivant les mots, que je traduis, de Ngugi Wa Thiongo, son premier directeur, «le centre est le modèle d'un monde de libre conversation entre les langues marginalisées, et entre toutes ces langues et celle qui est actuellement dominante » (ICWT Newsletter, 3, 2, 2006). 
Les textes demeurés sous forme de feuilleton (fon) ou de livres confidentiels, comme en bambara, voire d'objets universitaires comme en wolof (avant Doomi Golo) ne sauraient contribuer à cette conversation entre les langues que Ngugi appelle de ses vœux. Lui-même a publié en gikuyu, au Kenya, en trois tomes, les six livres de son roman. Nous savons qu'il est en exil aux Etats Unis et qu'il mène un combat politique et culturel. Les romans peuvent-ils demeurer des outils expérimentaux ? Le livre de Ngugi est aussitôt traduit en anglais chez un grand éditeur sous le titre de Wizard of the Crow (a translation from gikuyu by the author). L'original, Murogi wa kagogo, comprend six livres, répartis sur trois tomes, chaque livre étant consacré à un démon particulier: power, queuing, female, male, rebel, bearded. Le livre a été présenté à Nairobi le 15 janvier 2007 avec le dernier volume de la trilogie originale. Le Time (cité sur le site EAEP (East African Educational Publishers) le considère comme l'un des dix meilleurs livres de l'année; il a été écrit en gikuyu, et c'est certainement le plus gros livre écrit dans une langue africaine, si l'on excepte la Bible (qui n'est pas un texte original), selon la brochure de son éditeur kenyan.

La diaspora et l'exil peuvent-ils permettre à des littératures en des langues mineures de survivre sur Internet? Ne risque-t-on pas de faire une littérature hors sol, détachée des conditions sociales de pratique de la langue ? L'avenir du roman en tant que genre est aussi en jeu dans ces questions.

Ces textes nouveaux, ces fictions qui sont des innovations demandent à être traduits, commentés, édités. Ils posent la vaste question de l'historiographie des littératures de l'Afrique, particulièrement insuffisante et trop pénétrée de considérations politiques et diplomatiques. Il suffit de noter la diffusion d'une historiographie «francophone» qui fait comme si les autres langues n'existaient pas en Afrique. Cela est bien connu, mais doit être constamment répété. La littérature comparée est à cet égard une excellente médecine, trop peu pratiquée. Les textes fondateurs du roman africain, Moeti oa Bochabela, Bwana Myombekere na Bibi Bugonok, doivent être discutés dans le cadre du débat sur l'histoire littéraire de l'Afrique et non pas en tant que documents ethnologiques.

\section{RÉFÉRENCES BIBLIOGRAPHIQUES}

Bakhtine, Mikhail. Esthétique de la création verbale. Paris : Gallimard, 1984. 
Barthes, Roland. La préparation du roman. Paris : Le Seuil/Imec, 2003.

Bertoncini, Elena. "Reported Speech in Swahili literature », in J.Bloemaert (ed.), Swahili studies, Essays in Honour of Marcel Van Spaandonck. Ghent : Academia Press, 1991.

Booth, Wayne. The Rhetoric of fiction. Chicago : 1961.

Diop, Boris Boubacar [graphisé Joob, Bubakar Boris], Doomi Golo. Dakar : Editions Papyrus Afrique, 2003 [380 p.]

Etiemble, René. Essais de littérature (vraiment) générale. Paris : Gallimard, 1974.

Fagerberg-Diallo, Sonja. "Milk and Honey: Developing Written Literature in Pulaar», Yearbook of Comparative and General Literature, 1995, 43, p.67-83.

Garnier, Xavier et Ricard, Alain (dir.). L'effet roman. Arrivée du roman dans les langues d'Afrique. Paris : CENEL, Université de Paris 12, Harmatttan, 2006.

Garvin, Paul. «Le rôle des linguistes de l'Ecole de Prague dans le développement de la norme linguistique », in Bédard, Edith et Maurais, Jacques (eds). La norme linguistique. Québec : CILF, 1983, p. 141-152.

Glissant, Edouard. Le Discours antillais. Paris : Le Seuil, 1981.

Hartwig, Charlotte et Gerald. «Aniceti Kitereza, A Kerebe Novelist», Research in African Literature, 1972, 3, 2, p.162-170.

Kitereza, Aniceti. Bwana Myombekere na Bibi Bugonoka na Ntulanalwo na Bulihwali. Dar es Salaam : Tanzania Publishing House, 1980 (Traduction française, Les enfants du faiseur de pluie, Le tueur de serpents. Paris : Harmattan/Unesco, 1996, 1999).

Kunene, Daniel. Thomas Mofolo and the Emergence of Written Sesotho Prose. Johannesburg: Ravan Press, 1989.

Livre d'or de la Mission du Lesotho. Paris : Maison des Missions Evangéliques, 1912, [693 p.]

Maloka, Edward Tshidiso. Basotho and the Migrants : Towards a History of Labour Migrancy, 1890-1940. University of Cape Town, thèse de doctorat, 1995 [397 p.]

Mbelolo Ya Mpiku. «Introduction à la littérature kikongo », Research in African Literatures, 1972, 3, 2, p.117-161.

Mofolo, Thomas. Moeti Oa Bochabela. Morija, 1907 (Traduction française, L'Homme qui marchait vers le soleil levant. Bordeaux : Confluences, 2003).

Motsamai, Edouard et Machobane, James. Au temps des cannibales suivi de, Dans les cavernes sombres (traduits du sesotho). Bordeaux : Confluences, 1999 [171 p.]

Ngugi Wa Thiongo. Decolonizing the mind. Londres : J.Currey, 1986.

- - . Wizard of the Crow. Londres : Harvil Secker, 2006 [768 p.]

- - . Murogi wa Kagogo, Mbuku ya Mbere na ya keri. Nairobi : EAEP, 2004 [324 p. (livres un et deux)].

—-. Murogi wa Kagogo, Mbuku ya gatatu. Nairobi : EAEP, 2006 [225 p. (livre trois)]. 
- -. Murogi wa Kagogo, Mbuku ya kana, Gatano na Gatandatu. Nairobi : EAEP, 2007 [344 p. (livres quatre, cinq et six)].

Nossiter, Jonathan. Mondovino, dvd, 2004.

Pavel, Thomas. La pensée du roman. Paris : Gallimard, 2003.

Ricard, Alain. Littératures d'Afrique noire, des langues aux livres. Paris : Karthala/CNRS, 1995.

Riesz, János. De la littérature coloniale à la littérature africaine, prétextes, contextes, intertextes. Paris : Karthala, 2007.

Sissao, Alain. A la recherche du premier roman ou du premier récit en moore. LLACAN, ms, sl, 2003 [17 p.].

Sundkler, Bengt. Bantu Prophets in South Africa. Londres : Lutterworth Press, 1948.

Tshisungu wa Tshisungu, José. La littérature congolaise écrite en ciluba. Sudbury: Editions Glopro, 2006.

Vachek, Josef. A Prague School Reader in Linguistics. Bloomington : Indiana University Press, 1964.

\section{ANNEXE : PREMIERS ROMANS EN LANGUES DE L'AFRIQUE AU XXE SIECLE}

1904 [malgache] : Rabary, Raketaka Zandikro [Raketaka ma petite sœur], (feuilleton paru dans Ny Mpanolo-tsaina).

1907 [sesotho] : T. Mofolo, Moeti Oa Bochabela [L'homme qui marchait vers le soleil levant], $160 \mathrm{p}$.

1907 [xhosa] : Samuel Mqayi, Usamson, Lovedale Mission Press, 25 p.

1908 [amharique] : Afa Wark, Lebb Wallad Tarik [Récit venu du cœur], Rome, 90 p.

1913 [akan] : J. J. Adaye, Bere Adu [Le moment est venu], Akropong, B. M. Press, 90 p.

1930 [yoruba]: I. Thomas, Itan Igbesi Aye Emi Segilola Eleyinju-Ege [Récit de ma vie à moi, Segilola, aux yeux aguichants, qui ai eu mille maris].

1932 [boulou] : Louis Njemba Medou, Nnanga Kon, Ebolowa, Hasey Memorial Press.

1933 [zoulou] : John Langalibalele Dube, Insila ka Tshaka, [Le bras droit de Chaka»], Marianhill, Mission Press, 80 p.

1933 [igbo] : Pita Mwana, Omenuko, London, 94 p.

1934 [haoussa] : 1. Abubakar Imam, Ruwan Bagaja ; 2. Bello Kagara, Gan॰oki ; 3. Abubakar Tafawa Balewa, Shaihu Umar; 4. Muhammadu Gwarzo, Idon Matambayi; 5. John Tafida et Rupert East, Jiki Magayi.

1934 [kiswahili]: James Mbotela, Uhuru wa Watumwa [La libération des esclaves], Sheldon Press, London, $88 \mathrm{p}$.

1945 [kikerewe] : Aniceti Kitereza, Myombekere na Bugonoka na Ntulanalwo na Bulihwali [M et B, leur fils $\mathrm{N}$ et leur fille B] (inédit en kikerewe, publié en kiswahili en 1980, Dar es Salaam, Tanzania Publishing House, 593 p).

1946 [gikuyu/nouvelle] : Gakaara Wa Wanjau, Uhoro wa Ugurani [Histoire d'un mariage], $\mathrm{ABW}, 15 \mathrm{p}$.

1949 [ewe] : Sam Obianim, Amegbetoa alo Agbezuge fe nutinya [Amegbetoa ou les aventures d'Agbezuge], Ho, E.P.Church Press. 
1954 [luganda]: Edward K. N. Kawere, Zinunula Omunaku, Kampala, Uganda Literature Bureau.

1956 [shona] : Solomon Mutswairo, Feso, Cape Town, Oxford University Press in association with the Southern Rhodesian African Literature Bureau.

1956 [ndebele] : Ndabaningi Sithole, AmaNdebele kaMzilikazi [Les Ndebele de Mzilikazi].

1962 [kinyarwanda] : Simon Munyakazi, Ntabajyana, Amitiés belgo-rwandaises.

1963 [kabyle] : Belaid At-Ali, Lwali n Wedrar [1945], dans Dallet J.M. et J. L. Degezelle, Les Cahiers de Belaid ou la Kabylie d'antan, (I. Textes), FDB, Fort-National.

1967 [somali/nouvelles] : 1. Maxamed Faarax Cabdillaahi, Dhiif iyo jacayl [Désastre et Amour] ; 2. Axmed Cartan Xaange, Qawdhan iyo Qoran [Qawdhan et Qoran].

1974 [somali] : Faarax M.J. Cawl, Aqoondarro waa u nacab jacayl [L'ignorance est l'ennemie de l'amour].

1976 [lingala] : Yoka Mampunga, Makalamba [publication confidentielle (20 ex.) en 1966], Kinshasa, éd. Bobiso, $168 \mathrm{p}$.

1978 [gikuyu]: Lawrence Mwema, Mwana Mwagi [Un pauvre garçon], Nairobi, Kenya Literature Bureau, $83 \mathrm{p}$.

1981 [peul] : Yero Dooro Jallo, Darol Ndikkiri Jom Molo [Histoire de Ndikkiri le guitariste, joueur de moolo], Kawtal jangove pular (fulfulde) e leyze arabeve to Qahira, Le Caire, 1981, $116 \mathrm{p}$.

1981 [fon ] : Bo gbe die a? [Quelle vie !], Alidenu Vignonde (Jean-Norbert Vignonde), Paris, Binndi e Jande.

1983 [oromo] : Gaaddisaa Birruu, Kuusaa Gadoo [La rancune accumulée], Ethiopia (s.l.).

1992 [wolof] : Maam Yunus JED, Aawo bi, Ndakaaru, IFAN Cheikh Anta Diop, 1992, 72 p.

1996 [bambara] : Samba Niaré, Kanuya Wale [Un acte d'amour], Bamako, Somed, 163 p.

1998 [ciluba] : Pius Ngandu Nkashama, Bidi ntwilu, bidi mpelelu, Bruxelles-Lubumbashi, éd. Impala-Saint-Paul, $188 \mathrm{p}$. 